\title{
A diagram to determine the evaporation status of extrasolar planets ${ }^{\star}$
}

\author{
A. Lecavelier des Etangs \\ Institut d'Astrophysique de Paris, CNRS, UMR7095: Université Pierre et Marie Curie-Paris6, 98bis boulevard Arago, \\ 75014 Paris, France \\ e-mail: lecaveli@iap.fr
}

Received 13 February 2006 / Accepted 26 September 2006

\section{ABSTRACT}

\begin{abstract}
Aims. To describe the evaporation status of extrasolar planets, we consider an energy diagram in which the potential energy of the planets is plotted versus the energy received by the upper atmosphere.

Methods. Here we present a basic method to estimate these quantities. For the potential energy, we include the modification of the gravity field by the tidal forces from the parent stars.

Results. This description allows a rapid estimate of both the escape rate of the atmospheric gas and the lifetime of a planet against the evaporation process. In the energy diagram, we find an evaporation-forbidden region in which a gaseous planet would evaporate in less than 5 billion years. With their observed characteristics, all extrasolar planets are found outside this evaporation-forbidden region. The escape rates are estimated to be in the range $10^{5} \mathrm{~g} \mathrm{~s}^{-1}$ to $10^{12} \mathrm{~g} \mathrm{~s}^{-1}$, with a few cases above $10^{11} \mathrm{~g} \mathrm{~s}^{-1}$. The estimated escape rate for HD $209458 \mathrm{~b}$ is consistent with the lower limit of $10^{10} \mathrm{~g} \mathrm{~s}^{-1}$ obtained from interpretation of the H I Lyman- $\alpha$ observations.

This diagram suggests possibilities for the nature of the recently discovered Neptune-mass planets. We find that GJ $436 \mathrm{~b}, 55 \mathrm{Cnc}$ e and HD $69830 \mathrm{~b}$ cannot be low mass gaseous planets. With a density that must be above $0.5 \mathrm{~g} \mathrm{~cm}^{-3}$ to survive evaporation, these planets must contain a large fraction of solid/liquid material. We find that GJ $876 \mathrm{~d}$ must have a density greater than $\sim 3 \mathrm{~g} \mathrm{~cm}^{-3}$ to survive the strong EUV energy flux from its nearby parent star. GJ $876 \mathrm{~d}$ must contain a large fraction of massive elements.
\end{abstract}

Key words. stars: planetary systems

\section{Introduction}

Among the nearly two hundreds extrasolar planets so far identified, there is a particular class of planets very close to their parent stars with semi major axis $\lesssim 0.1 \mathrm{AU}$. Among them, there are massive hot-Jupiters (also named "Pegasides", Guillot et al. 1996) and small mass planets (see for instance, Santos et al. 2004; Rivera et al. 2005). These planets constitute about $20 \%$ and $5 \%$ respectively, of the planets identified today. Since the discovery of the first of them, 51 Peg b (Mayor \& Queloz 1995), the issue of their evaporation status has been raised (Burrows \& Lunine 1995; Guillot et al. 1996). Since these planets exist and orbit stars that are not particularly young, the evaporation rate of the observed massive hot-Jupiters should be modest enough to not dramatically impact their evolution. They must have survived at least billion years at the place where they are observed. It is extremely unlikely that we are witnessing a transient phase in the evolution of extrasolar planets (Baraffe et al. 2004). Thus, the discovery of a large number of massive hot-Jupiters ${ }^{1}$ and very-hot-Jupiters ${ }^{2}$ led to the conclusion that the evaporation of massive planets has to be modest.

In that frame the discovery that the transiting extrasolar planet HD 209458b is indeed losing mass was unexpected (Vidal-Madjar et al. 2003). From the observation

\footnotetext{
* Appendices A-C are only available in electronic form at http://www. aanda.org

1 Usually defined as massive planets orbiting in less than 10 days.

${ }^{2}$ Usually defined as planets orbiting in less than 3 days (e.g., Gaudi et al. 2005).
}

of $15 \pm 4 \%$ absorption in Lyman- $\alpha$ during the transit, a lower limit of the HI escape rate was estimated to be of the order of $\sim 10^{10} \mathrm{~g} \mathrm{~s}^{-1}$ (Vidal-Madjar et al. 2003; Vidal-Madjar \& Lecavelier des Etangs 2004). This mass loss rate corresponds to a loss of $\sim 0.1 \%$ of the total mass of the planet over 10 billion years $(10 \mathrm{Gyr})$. Models of the time variation of this escape rate lead to slightly larger values for the total fraction of mass lost over the whole stellar life (see e.g., Lecavelier des Etangs et al. 2004; Baraffe et al. 2004).

Different detailed models have been developed to understand the observed evaporation process and to estimate the escape rate (e.g., Yelle 2004; Tian et al. 2005; García Muñoz 2006), to be compared with the observational constrains (Vidal-Madjar et al. 2003, 2004). It has been also suggested that evaporation can lead to significant modification of the planets' nature in the case of planets with a mass only a fraction of the mass of the observed hot-Jupiter and orbiting close to their parent stars (Lecavelier des Etangs et al. 2004; Baraffe et al. 2005).

However, models were applied to specific cases; it is not yet possible to conclude on the order of magnitude of the possible evaporation of planets that are being discovered, without a detailed model for each particular case. The purpose of the present paper is not to review previous works and extended modeling efforts. Here we propose an alternative approach to obtain the general characteristics from the basic observed parameters. This approach allows statistical conclusions without the need to model in detail each individual planet for which detailed physical characteristics may not be available. We outline the main characteristics of a given planet that allow a conclusion on the general 
trend of its evaporation status, escape rate and lifetime related to the erosion through evaporation.

Here we present the idea of an energy diagram to address these issues (Sect. 2), and the way to simply estimate the energetic characteristics of a given planet (Sects. 3 and 4). An evaluation of the lifetime and escape rates is presented in Sect. 5. The resulting diagram is shown in Sect. 6. A diagram corresponding to the escape rate is given in Sect. 7. Uncertainties and evolutionary tracks are presented in Sects. 8 and 9. We discuss the possible nature of the recently discovered Neptune mass planets in Sect. 10.

\section{Potential energy of planets versus stellar energy flux}

A plot of the lifetime of planets as a function of their masses and orbital distances has been provided by Lecavelier des Etangs et al. (2004). This plot shows that the identified planets are stable against evaporation. For the existing planets, the lifetime, defined as the time to escape the full mass of the planet, is always longer than $\sim 10^{10}$ years (Fig. 5 of Lecavelier des Etangs et al. 2004). Attempts have been made to extrapolate from this plot the possible escape rate in the case of planets discovered later (HD 149026 b, Valenti 2005). It would be valuable to have a rapid method to determine the evaporation rate of a given planet to predict if we might observe and constrain the evaporation through, e.g., Lyman- $\alpha$ observations during a transit (Lecavelier des Etangs et al., in preparation). However, in the lifetime plot of Lecavelier des Etangs et al. (2004), the energy flux from the star is assumed to be that of HD 209458 b. The energy flux from other stars is different depending on the stellar type, rotation period, etc. The second critical parameter in this plot is the assumed planetary radii. In the lifetime plot of Lecavelier des Etangs et al. (2004), the radius of HD 209458 b (1.35 Jupiter radii) was used to normalize the scaling laws for the planetary radii because at that time HD 209458 b was the only hot-Jupiter whose radius had been measured. However, the radius of HD 209258 b is now considered as anomalously large (Guillot 2005; Lecavelier des Etangs \& Vidal-Madjar 2006). From observations of ten transiting planets (Bouchy et al. 2005b; McCullogh et al. 2006) and detailed modeling of the planetary structure (Guillot 2005), 1.1 Jupiter radii is considered more appropriate for a 1 Jupiter mass planet close to its parent star. This makes HD 209458 b with its 1.35 Jupiter radii and 0.69 Jupiter mass a peculiar object. In the following we will therefore use a fit to Guillot's model. A new plot has been made with this new information (Lecavelier des Etangs \& Vidal-Madjar 2006). However, the planetary radius depends also on parameters other than the planetary mass and orbital distance, for instance, the stellar type and the corresponding effective temperature of the central star and its age. It is thus impossible to uniquely estimate the lifetime and escape rate from the position of a given planet in the orbital distance versus mass diagram. As a consequence, this plot has to be revisited.

Thus, since each planet has a different radius and different energy flux entering the upper atmosphere from stars of different types, the orbital distance versus mass diagram is not appropriate to conclude on the escape rate and life time of a variety of planets. We have to define the parameters (or combination of parameters) that allow us to determine these escape rate and life time.

After the realization that HD 209458 b is losing mass, many modeling efforts have been pursued to better understand escape in the particular case of hot-Jupiters. The common result of these models is that almost $100 \%$ of the EUV/Lyman- $\alpha$ energy flux that is converted into heat is then transferred into escape (see, for instance, Yelle 2004; and Tian et al. 2005). Even magnetic fields that can inhibit ion escape are found to have modest impact. Yelle (2004) found that the net escape rate with ion escape inhibited by the magnetic field is only $30 \%$ smaller than in its reference model. Similarly, the effects of dissociation and ionization of $\mathrm{H}_{2}$ are found to be negligible. The resulting escape rates of these various models are different not because these models assumed different mechanisms but because of the different illumination, planetary cross section for the energy flux, and fraction of this energy converted into heat in the upper atmosphere.

As a result, the evaporation status of a set of different planets orbiting different stars can be obtained by a comparison of the energy deposited in the upper atmosphere and used to escape the planet gravity, i.e. to compensate for the (negative) potential energy of the atmospheric gas with this potential energy. We therefore propose to calculate the position of the extrasolar planets in a diagram of potential energy versus the stellar energy. We now evaluate these two quantities in the two next sections to plot the corresponding diagrams in Sects. 6 and 7.

\section{The extreme ultraviolet illumination from the central stars}

The present and past illumination from the parent stars is a key parameter. First, as the planet-hosting stars are cooler than early $\mathrm{F}$ stars in the main sequence, the stellar energy emitted in the near UV range is negligible (e.g., Kurucz 1993). Moreover, near UV and optical energy is absorbed deep in the bottom and dense atmosphere. On the contrary, neutral components of atmospheres have large cross section in the extreme ultraviolet (100 $\AA<\lambda<1200 \AA)$. Photons in the extreme ultraviolet wavelength range (EUV) are thus easily absorbed by the tenuous upper atmospheres of planets. For instance, in the terrestrial planets of the Solar system, the EUV energy flux is the main driver of the escape (e.g., Chamberlain \& Hunten 1987). Therefore, for simplicity and in agreement with the results of all detailed models of the evaporation of hot-Jupiters, we can assume that the energy flux deposited in the upper atmosphere and transferred into escape energy mainly comes from the EUV.

However, because of interstellar absorption, there are very few observational constraints on the EUV flux from the stars in the solar neighborhood. On the other hand, although stellar EUV fluxes are related to stellar rotational velocities (Wood et al. 1994), most of the stars harboring extrasolar planets have no published rotational velocities or only upper limits. In most cases, information on the planet-hosting stars is limited to the stellar type. We have hence chosen to consider the very extreme ultraviolet luminosities $L_{\mathrm{S} 2}$ as a function of the stellar type, as measured by Rosat in the S2 channel between 110 and $200 \AA$. We then scale these luminosities to the solar EUV flux of $\sim 4.6 \mathrm{erg} \mathrm{cm}^{-2} \mathrm{~s}^{-1}$ at $1 \mathrm{AU}$ as given by Ribas et al. (2005). This solar value is obtained considering mid-solar cycle data of 1993 as representative of the Sun at average activity. The EUV flux, $F_{\text {EUV }}(1 \mathrm{AU})$, received by a planet per unit area at $1 \mathrm{AU}$ is hence calculated using

$F_{\mathrm{EUV}}(1 \mathrm{AU})=4.6 \frac{L_{\mathrm{S} 2}}{L_{\mathrm{S} 2, \mathrm{G} \text { stars }}} \mathrm{erg} \mathrm{cm}^{-2} \mathrm{~s}^{-1}$.

The scale between $L_{\mathrm{S} 2}$ luminosities and EUV is justified by the fact that both the $\sim 100-200 \AA$ and the extreme-far UV fluxes are 
emitted by the same region in the chromosphere and the transition region where the temperature is observed to increase from the photospheric to the coronal values.

Hodgkin \& Pye (1994) give median S2 luminosities as a function of the spectral types, with value of $10^{27.9}, 10^{26.8}, 10^{27.3}$ and $10^{26.6} \mathrm{erg} \mathrm{s}^{-1}$ for $\mathrm{F}, \mathrm{G}, \mathrm{K}$ and $\mathrm{M}$ stars respectively. Most of the $\mathrm{F}$ stars known to harbor planets are late type $\mathrm{F} 8 \mathrm{~V}$ or F9V stars. Because early F stars remain chromospherically active and fast-rotating during their main sequence phase, the accuracy in the measurement of their radial velocity is strongly limited. It is still impossible to detect planets around them (F. Pont, private communication). But these early $F$ stars contribute to the mean value of the observed S2 luminosities of the whole class of F stars. We therefore decided to consider a typical S2 luminosity of $10^{27.3} \mathrm{erg} \mathrm{s}^{-1}$ for F6 and F7 stars harboring planets, and an S2 luminosity of $10^{26.8} \mathrm{erg} \mathrm{s}^{-1}$ for later F8V and F9V stars which are more similar to G0V type stars.

Using these S2 luminosities and the solar EUV luminosity, we find $F_{\text {EUV }}(1 \mathrm{AU})=14.7,4.6,4.6,14.7$ and $2.9 \mathrm{erg} \mathrm{cm}^{-2} \mathrm{~s}^{-1}$ for F6-F7, F8-F9, G, K, and M stars, respectively. Except if explicitly mentioned (Sect. 8), these values will be used throughout the paper. These fluxes can be converted to the energy per unit of time, $\mathrm{d} E_{\mathrm{EUV}} / \mathrm{d} t$, received by a planet of radius $R_{\mathrm{p}}$ with an orbital distance $a_{\mathrm{p}}$ using:

$\mathrm{d} E_{\mathrm{EUV}} / \mathrm{d} t=\pi R_{\mathrm{p}}^{2}\left(\frac{a_{\mathrm{p}}}{1 \mathrm{AU}}\right)^{-2} F_{\mathrm{EUV}}(1 \mathrm{AU})$.

\section{Potential energy}

To evaluate the total lifetime of a planet from an energetic point of view, we need to consider the total potential energy, $E_{\mathrm{p}}$, of the planet considered as a whole, from its surface to its deep interior. If this energy is given in erg, the ratio with the received energy expressed in erg per billion years will provide an estimate of the total time needed to cancel the (negative) potential energy, in other words, the evaporation lifetime. To evaluate the escape of the atmospheric gas at the present time, we need to consider the potential energy of the gas only in the atmosphere and per unit mass. The ratio of this quantity to the energy received will provide the mass escape rate. We have two different quantities that need to be estimated: the potential energy of the whole planet and the potential energy per unit of mass in the atmosphere.

\subsection{The potential energy of the whole planet}

The potential energy of a gaseous sphere of radius $R_{\mathrm{p}}$ is given by

$$
E_{\mathrm{p}}=-G \int_{0}^{R_{\mathrm{p}}} \frac{m(r)}{r} \frac{\mathrm{d} m(r)}{\mathrm{d} r} \mathrm{~d} r
$$

where $G$ is the gravitational constant and $m(r)$ is the mass inside the radius $r$. For a uniform density and a mass $M_{\mathrm{p}}$, we obtain the classical formula $E_{\mathrm{p}}=-3 G M_{\mathrm{p}}^{2} / 5 R_{\mathrm{p}}$. In general, the potential energy depends on the internal structure. For a planet with a mass $M_{\mathrm{p}}$ and a radius $R_{\mathrm{p}}$, an analytical approximation can be found assuming a polytropic approximation of the equation of state for the planet interior. Using a polytropic index $n=1$ $\left(P \propto \rho^{2}\right.$, de Pater \& Lissauer 2001) the potential energy is found to be:

$E_{\mathrm{p}}=-\frac{3}{4} G M_{\mathrm{p}}^{2} / R_{\mathrm{p}}$
The details of the calculation are given in Appendix A. Numerically, this converts into:

$E_{\mathrm{p}}=-2.9 \times 10^{43}\left(\frac{M_{\mathrm{p}}}{M_{\text {Jup }}}\right)^{2}\left(\frac{R_{\mathrm{p}}}{R_{\text {Jup }}}\right)^{-1} \mathrm{erg}$.

\subsection{The potential energy per unit of mass in the atmosphere}

Another interesting quantity is the potential energy of the atmospheric gas per unit mass. This potential energy per unit of mass is simply given by (see Appendix B):

$\mathrm{d} E_{\mathrm{p}(\mathrm{atm})} / \mathrm{d} m=-G M_{\mathrm{p}} / R_{\mathrm{p}}$.

Numerically, this converts into:

$\mathrm{d} E_{\mathrm{p}(\mathrm{atm})} / \mathrm{d} m=-1.9 \times 10^{13}\left(\frac{M_{\mathrm{p}}}{M_{\text {Jup }}}\right)\left(\frac{R_{\mathrm{p}}}{R_{\mathrm{Jup}}}\right)^{-1} \mathrm{erg} \mathrm{g}^{-1}$.

The potential energy per unit of mass of the atmospheric gas is much higher than its kinetic or thermal energy, even if the gas is escaping at high velocity. For instance, even for gas escaping with the high velocity of $10 \mathrm{~km} \mathrm{~s}^{-1}$, the kinetic energy is $5 \times 10^{11} \mathrm{erg} \mathrm{g}^{-1}$, compared to the typical value of $-\mathrm{d} E_{\mathrm{p}(\mathrm{atm})} / \mathrm{d} m \sim$ $10^{13} \mathrm{erg} \mathrm{g}^{-1}$ for the known extrasolar planets (Sect. 7). Even for very-hot-Jupiters in which tidal forces play a significant role (Sect. 4.3), the potential energy is expected to be always greater than the kinetic energy. The kinetic energy of the escaping gas is thus neglected in the present analysis.

\subsection{Potential energy and tidal forces}

In the case of hot-Jupiters and very-hot-Jupiters, the gravity from the nearby parent star modifies the geometry of the potential energy. In those cases, the iso-potentials around the planet at high altitude are no longer spherical and are open to infinity when the potential reaches the value of the Roche lobe. Therefore the energy needed to escape the planet can be defined by the energy needed to reach this Roche lobe. In the case of $M_{\mathrm{p}} \ll M_{*}$, the potential energy of the atmospheric gas per unit mass is accordingly modified by

$\mathrm{d} E_{\mathrm{p}(\mathrm{atm})}^{\prime} / \mathrm{d} m=\mathrm{d} E_{\mathrm{p}(\mathrm{atm})} / \mathrm{d} m+\Delta_{\text {tidal }} \mathrm{d} E_{\mathrm{p}(\mathrm{atm})} / \mathrm{d} m$,

where (see Appendix B)

$\Delta_{\text {tidal }} \mathrm{d} E_{\mathrm{p}(\mathrm{atm})} / \mathrm{d} m \approx \frac{3^{4 / 3}}{2} \frac{G M_{*}^{1 / 3} M_{\mathrm{p}}^{2 / 3}}{a_{\mathrm{p}}}$,

$a_{\mathrm{p}}$ being the orbital distance of the planet and $M_{*}$ the mass of the central star.

The potential energy of the whole planet as defined in Sect. 4.1 is also modified by tidal forces. We can define $\Delta_{\text {tidal }} E_{\mathrm{p}}$ by

$E_{\mathrm{p}}^{\prime}=E_{\mathrm{p}}+\Delta_{\mathrm{tidal}} E_{\mathrm{p}}$.

Because Eq. (9) does not depend on the planetary radius, the change introduced by the tidal forces does not depend on the planet's internal structure. We have

$\Delta_{\text {tidal }} E_{\mathrm{p}}=\int_{0}^{R_{\mathrm{p}}} \frac{3^{4 / 3}}{2} \frac{G M_{*}^{1 / 3} m(r)^{2 / 3}}{a_{\mathrm{p}}} \frac{\mathrm{d} m(r)}{\mathrm{d} r} \mathrm{~d} r$.

For the total potential energy of a planet we have

$\Delta_{\text {tidal }} E_{\mathrm{p}} \approx \frac{3^{7 / 3}}{10} \frac{G M_{*}^{1 / 3} M_{\mathrm{p}}^{5 / 3}}{a_{\mathrm{p}}}$ 
Because $\Delta_{\text {tidal }} \mathrm{d} E_{\mathrm{p}(\mathrm{atm})} / \mathrm{d} m$ is positive, the energy deposition needed to escape the planet's gravity is decreased by the tidal forces. For the identified hot-Jupiters, we find that the depth of their potential well is decreased by the tidal forces by typically 30 to $50 \%$. For instance, we find that $\Delta_{\text {tidal }} \mathrm{d} E_{\mathrm{p}(\mathrm{atm})} /\left|\mathrm{d} E_{\mathrm{p}(\mathrm{atm})}\right| \sim 50 \%$ for OGLE-TR-56b and $\sim 33 \%$ for HD 209458 b. Keeping the hypothesis that the escape energy is mainly used to escape the potential well of the planet, this leads to a similar increase of the escape rate (Sect. 5).

\section{Lifetime and escape rate}

Assuming that the energy deposited by the EUV flux is used to extract the gas that escapes the potential well of the planet with an efficiency $\epsilon=1$, we can derive the escape rate, $\mathrm{d} m / \mathrm{d} t$, and a corresponding typical lifetime for a given planet. Assuming $-\mathrm{d} E_{\mathrm{p}(\mathrm{atm})}^{\prime}=\epsilon \mathrm{d} E_{\mathrm{EUV}}$, and neglecting the tidal forces $\left(\mathrm{d} E_{\mathrm{p}(\mathrm{atm})}^{\prime} \approx\right.$ $\left.\mathrm{d} E_{\mathrm{p}(\mathrm{atm})}\right)$, we find:

$\mathrm{d} m / \mathrm{d} t=-\frac{\mathrm{d} E_{\mathrm{EUV}} / \mathrm{d} t}{\mathrm{~d} E_{\mathrm{p}(\mathrm{atm})} / \mathrm{d} m}$.

From Eq. (2), we derive the escape rate

$\mathrm{d} m / \mathrm{d} t=F_{\mathrm{EUV}}(1 \mathrm{AU})\left(\frac{\pi R_{\mathrm{p}}^{3}}{G M_{\mathrm{p}}}\right)\left(\frac{a_{\mathrm{p}}}{1 \mathrm{AU}}\right)^{-2}$.

Numerically, this gives

$$
\begin{aligned}
\mathrm{d} m / \mathrm{d} t= & 3.7 \times 10^{7}\left(\frac{F_{\mathrm{EUV}}(1 \mathrm{AU})}{4.6 \mathrm{erg} \mathrm{cm} \mathrm{cm}^{-2} \mathrm{~s}^{-1}}\right) \\
& \times\left(\frac{R_{\mathrm{p}}}{R_{\mathrm{Jup}}}\right)^{3}\left(\frac{M_{\mathrm{p}}}{M_{\mathrm{Jup}}}\right)^{-1}\left(\frac{a_{\mathrm{p}}}{1 \mathrm{AU}}\right)^{-2} \mathrm{~g} \mathrm{~s}^{-1} .
\end{aligned}
$$

We can similarly assume $-E_{\mathrm{p}}^{\prime}=\epsilon \int_{0}^{t 1} \mathrm{~d} E_{\mathrm{EUV}}$ to obtain the typical duration, $t_{1}$, which corresponds to the time needed to fill the whole potential well of the planet assuming the current energy flux. This duration can hence be called a "lifetime"; the nature of a planet can change through evaporation if $t_{1}$ is shorter than the typical lifetime of the parent star. If the energy received by the planet, $\mathrm{d} E_{\mathrm{EUV}} / \mathrm{d} t$, is assumed to be constant over time, from the above equation we derive the lifetime $t_{1}$ :

$t_{1}=\frac{-E_{\mathrm{p}}^{\prime}}{\mathrm{d} E_{\mathrm{EUV}} / \mathrm{d} t}$.

However, when the planetary mass evolves, the radius and the corresponding energy flux captured by the planet changes; the real lifetime also changes. A more detailed analysis is possible by considering the variations of the escape rate as a function of time. This leads to a new "lifetime", $t_{2}$, which can be defined by

$-E_{\mathrm{p}}^{\prime}=\left(\frac{a_{\mathrm{p}}}{1 \mathrm{AU}}\right)^{-2} \int_{t_{0}}^{t_{2}} \pi R_{\mathrm{p}}\left(t^{\prime}\right)^{2}\left(F_{\mathrm{EUV}}(1 \mathrm{AU})\left(t^{\prime}\right)\right) \mathrm{d} t^{\prime}$,

where $t_{0}$ is the initial time of the system. At this stage a useful quantity is the mean value of the EUV energy flux from the central star $\left\langle\mathrm{d} E_{\mathrm{EUV}} / \mathrm{d} t\right\rangle$ defined by:

$$
\begin{aligned}
\left\langle\mathrm{d} E_{\mathrm{EUV}} / \mathrm{d} t\right\rangle(t) \equiv & \left(\frac{a_{\mathrm{p}}}{1 \mathrm{AU}}\right)^{-2} \\
& \times \frac{\int_{t_{0}}^{t} \pi R_{\mathrm{p}}\left(t^{\prime}\right)^{2}\left(F_{\mathrm{EUV}}(1 \mathrm{AU})\left(t^{\prime}\right)\right) \mathrm{d} t^{\prime}}{\left(t-t_{0}\right)},
\end{aligned}
$$

which includes the variations of the EUV flux and the variation of the planetary radius which both are larger during the early stage of the planetary system (Guinan \& Ribas 2002; Ribas et al. 2005; Burrows et al. 2000). We can define a correction factor for the time variation of the energy flux received by a planet,

$\gamma \equiv \frac{\left\langle\mathrm{d} E_{\mathrm{EUV}} / \mathrm{d} t\right\rangle}{\mathrm{d} E_{\mathrm{EUV}} / \mathrm{d} t}$

Hence we have

$t_{2} \approx \gamma^{-1} t_{1}$.

We now have to evaluate the impact of this $\gamma$ correction factor. The variation of the EUV flux as a function of time has been thoroughly analyzed for G stars by Ribas et al. (2005) within the "Sun in time" program. A recent work reports similar results for K type stars (Lakatos et al. 2005). According to these works, the EUV flux can be assumed to follow a power law in the form $F \propto t^{-\alpha}$, where $\alpha$ is close to 1. For $\mathrm{G}$ type stars, the 1-1200 A EUV flux is found to have $\alpha \sim 1.23$, while the Lyman- $\alpha$ has $\alpha=0.72$ (Ribas et al. 2005). For K type stars, the stellar C IV emission lines, which are believed to emerge from the same coronal region as the EUV flux, follows a power law with $\alpha \sim 0.94$. We therefore can consider a power law with $\alpha=1$ as a good approximation (see also Vardavas 2005). Assuming that the increase of the UV luminosities in the past must be limited and should "saturate", we consider that the luminosities do not vary at ages below 100 million years (Lakatos et al. 2005). With these values, and neglecting for the moment the variation of the planetary radius, we find that for $\alpha=1.23$ we have $\gamma=6.5$ at $t=5 \mathrm{Gyr}$, and for $\alpha=0.75$ we have $\gamma=4$. We can therefore consider a power law with $\alpha=1$ as a good approximation.

The variation of the planetary radius is more complex because this depends on the planetary characteristics like mass and orbital distance. Nonetheless, we found empirically that the published time variation of the planetary radius (Burrows et al. 2000; Guillot 2005) can be well fitted by a simple scaling law of the form:

$R_{\mathrm{p}}(t)=R_{\infty}+\beta t^{-0.3}$

We find good fits with $\beta=0.2 R_{\infty} \mathrm{Gyr}^{0.3}$ for $M_{\mathrm{p}} \gtrsim 0.3 M_{\mathrm{Jup}}$, and with $\beta=0.3 R_{\infty} \mathrm{Gyr}^{0.3}$ for $M_{\mathrm{p}} \approx 0.1 M_{\text {Jup }}$.

Using $\alpha=1$ for the power low of the luminosity variations and $\beta=0.2 R_{\infty} \mathrm{Gyr}^{0.3}$ for the variation of the planetary radius, we find $\gamma=6.8$ at $t=2 \mathrm{Gyr}$ (the typical age of the F6-F7 stars harboring planets, Saffe et al. 2005) and $\gamma=8.0$ at $t=5 \mathrm{Gyr}$ (taken as the typical age of later type stars). Using for less massive planets $\beta=0.3 R_{\infty} \mathrm{Gyr}^{0.3}$, we find $\gamma=8.7$ at $t=2 \mathrm{Gyr}$ and $\gamma=10.0$ at $t=5 \mathrm{Gyr}$. In the following we decided to take $\gamma=7$ for F6-F7 stars, and $\gamma=8$ for later type stars.

If we consider that the EUV luminosities can be extrapolated with the $t^{-1}$ function before the age of 100 million years, down to the age of 30 million years, $\gamma$ is then increased by about $25 \%$. We thus conclude that the correction factor for time variation of the energy flux does not strongly depend on the epoch of this "saturation" of the EUV luminosities in the past.

\section{The energy diagram}

With the equations derived in the previous sections, we can now plot diagrams of the potential energy versus energy flux (Fig. 1). In particular Eqs. (4) and (12) are used to derived the potential energy, $E_{\mathrm{p}}^{\prime}$, and Eq. (18) (or a combination of Eqs. (2) and (19)) 
is used to derive the mean EUV energy received per unit of time by the planets, $\left\langle\mathrm{d} E_{\mathrm{EUV}} / \mathrm{d} t\right\rangle$. We used the characteristics of the 182 identified planets as tabulated by Schneider (2006) on June 15th, 2006.

The only remaining difficulty in the drawing of the plot lies in the mass determination. Except for the case of transiting planets (Sect. 8), most of the planets have only a determination of $M_{\mathrm{p}} \sin i$, where $i$ is the orbit inclination. We compensate for this effect by multiplying the $M_{\mathrm{p}} \sin i$ by a factor $\sqrt{2}$. This $\sqrt{2}$ factor is taken because the real mass $M_{\mathrm{p}}$ is statistically smaller than $\sqrt{2} M_{\mathrm{p}} \sin i$ in $50 \%$ of the cases, and larger in $50 \%$ of the cases. The result is thus a spread of the position of the planets in the energy diagram. For transiting planets, because $\sin i \approx 1$, we keep the measured mass without applying a correction factor.

From these planetary masses and using the tabulated types of the parent stars we estimate the planetary radius by fitting the radius versus mass curves calculated by Guillot (2005). This model assumes a central rock core and a gaseous envelope following a hydrogen-helium equation of state with a helium mixing ratio $Y=0.30$. The estimated radii should not be sensitive to the evaporation, because they result from a calculation of the planet interior at several bars pressure not affected by the evaporation operating much higher in the atmosphere; this is confirmed by the absence of a correlation of the observed radii of transiting planets with age and EUV flux received during the planets' lifetime (Guillot et al. 2006). An indetermination remains for the very low mass planets (Neptune mass planets) because their radius (or density) as a function of their mass strongly depends on their nature. As a first approach and to plot the energy diagram in Fig. 1, we consider that these planets have a typical density of $2 \mathrm{~g} \mathrm{~cm}^{-3}$ for 15 Earth mass and $6 \mathrm{~g} \mathrm{~cm}^{-3}$ for 6 Earth mass. A detailed analysis of these planets in this diagram will be presented in Sect. 10.

The first plot (Fig.1) shows the total potential energy of the planet as a function of the mean energy flux $\left\langle\mathrm{d} E_{\mathrm{EUV}} / \mathrm{d} t\right\rangle$. We plot the mean energy flux on the abscissa axis in reverse order with larger values in the left. This keeps the planets with short orbital distances in the left part of the diagram for consistency with previous plots such as the diagram of Lecavelier des Etangs et al. (2004). The opposite of the potential energy, $-E_{\mathrm{p}}^{\prime}$, is larger for more massive planets, and massive planets are therefore found in the upper part of the diagram. This diagram is close to the classical diagram of mass versus orbital distance. However, in the present diagram the position reflects the impact of evaporation on the planetary nature.

We plot the diagram with the 182 identified extrasolar planets. The striking result is the absence of planets in two different regions of the diagram. The absence of planets in the bottom right region can be explained by the detection limit of the radial velocity surveys. We plotted the limit for a radial velocity of $10 \mathrm{~m} \mathrm{~s}^{-1}$. As expected, very few planets have radial velocities of the parent stars below this limit.

The most important region devoid of planets in the diagram is the bottom left region. This region should contain light planets at short orbital distances. However a plot of the lifetime line at $t_{2}=5 \mathrm{Gyr}$, shows that there are no planets in this part of the diagram because this is an evaporation-forbidden region. Planets in this region would receive more EUV energy than needed to fill the potential well of the planet and evaporate the gas, leaving a core, an evaporation remnant (also named a "chthonian" planet; Lecavelier des Etangs et al. 2004).

From the position in the diagram, the typical lifetime of a given planet can be extracted. If the mean energy

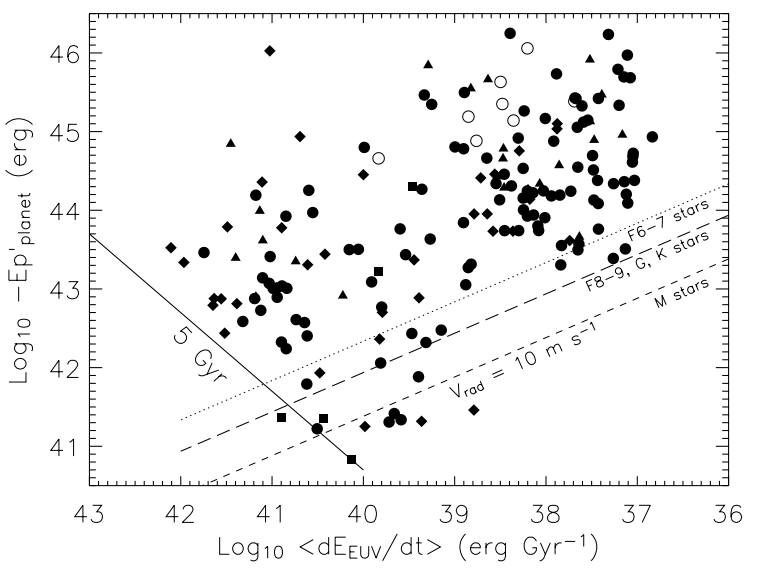

Fig. 1. Potential energy of the extrasolar planets as a function of the mean EUV energy received per billion years, $\left\langle\mathrm{d} E_{\mathrm{EUV}} / \mathrm{d} t\right\rangle$. The direction of the abscissa axis is with the largest value of the mean energy flux toward the left. The 182 identified planets are plotted with symbols depending on the type of the central star: triangles for F stars, filled circles for $\mathrm{G}$ stars, diamonds for $\mathrm{K}$ stars and squares for $\mathrm{M}$ stars; planets orbiting class III stars are plotted with empty circles. There are very few planets with radial velocities of the parent stars below the lines for which the radial velocity is $10 \mathrm{~m} \mathrm{~s}^{-1}$. The radial velocity iso-curves at $10 \mathrm{~m} \mathrm{~s}^{-1}$ have been plotted for various stellar types (see Appendix C); the iso-curves for F8-F9, G and K-type stars are nearly superimposed and are plotted with a single line. The striking result is the absence of planets in the bottom left region which corresponds to light planets (small $\left.-E_{\mathrm{p}}^{\prime}\right)$ at short orbital distances (large $\left.\left\langle\mathrm{d} E_{\mathrm{EUV}} / \mathrm{d} t\right\rangle\right)$. A plot of the lifetime line at $t_{2}=5 \mathrm{Gyr}$, shows that there are no planets in this part of the diagram simply because this is an evaporation-forbidden region. Planets in this region would receive more EUV energy than needed to fill the potential well of the planet, and evaporate in less than $5 \mathrm{Gyr}$.

flux $\left\langle\mathrm{d} E_{\mathrm{EUV}} / \mathrm{d} t\right\rangle$ is given in units of erg per billion years, and the potential energy is given in units of $e r g$, the simple ratio of both quantities provides the corresponding lifetime in billions of years. In the diagram, lifetime isochrones are straight lines. In Fig. 1, we plotted the line corresponding to the lifetime of $5 \mathrm{Gyr}$.

\section{Diagram of the potential energy of the atmosphere and the corresponding escape rate}

With the above equations (Eqs. (2), (6) and (9)) we can also plot the potential energy per unit mass of the atmosphere as a function of the present time energy flux (Fig. 2). We again superimposed the position of the 182 identified planets.

Although apparently similar to the previous diagram, this new diagram allows us to estimate the actual evaporation rate for a given planet, assuming that most of the energy in the extreme ultraviolet is used to escape the combined effect of planet gravity and tidal forces. In Fig. 1, we considered the total potential energy, providing a hint to the total escape mass and time. In Fig. 2, we consider the potential energy per unit of mass $\left(\mathrm{erg} \mathrm{g}^{-1}\right)$ of the atmospheric gas versus the present time input energy $\left(\mathrm{erg} \mathrm{s}^{-1}\right)$. The ratio of both quantities provides a hint to the present escape rate $\left(\mathrm{g} \mathrm{s}^{-1}\right)$.

From the plot we conclude that the extrasolar planets must have escape rate in the range $10^{5}$ to $10^{12} \mathrm{~g} \mathrm{~s}^{-1}$. The largest escape rates are obtained for OGLE-TR-113 b, HD $189733 \mathrm{~b}$, HD 46375 b, HD 63454 b, and TrES-1, for which we obtain escape rates of $3.5 \times 10^{11}, 3.2 \times 10^{11}, 2.8 \times 10^{11}, 2.5 \times 10^{11}$, and $2.2 \times 10^{11} \mathrm{~g} \mathrm{~s}^{-1}$, respectively. These five stars are cataloged 


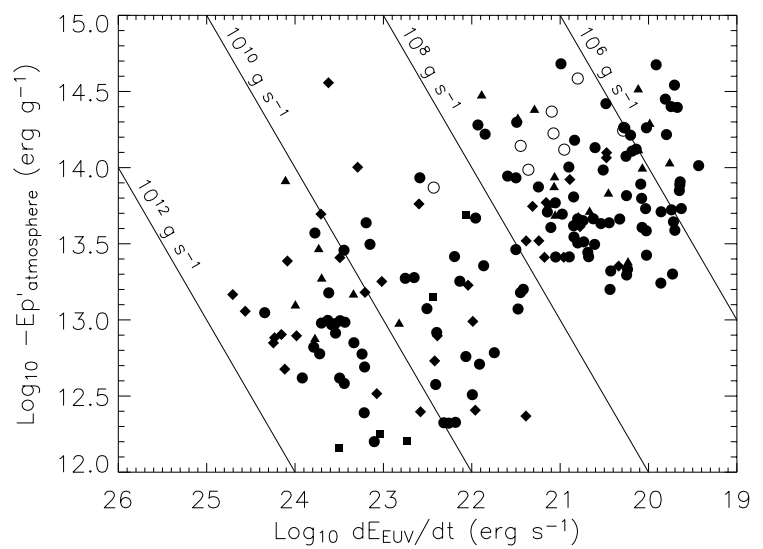

Fig. 2. The potential energy per unit of mass of the atmosphere of the extrasolar planets as a function of the EUV input flux. Unlike Fig. 1, the potential energy is per unit of mass at one planetary radius in $\mathrm{erg} \mathrm{g}^{-1}$; the EUV input flux is the value at the present time in $\mathrm{erg} \mathrm{s}^{-1}$. This allows us to directly estimate the corresponding escape rate at the present time in $\mathrm{g} \mathrm{s}^{-1}$. The escape rates are drawn with straight lines for $10^{6}, 10^{8}$, $10^{10}$ and $10^{12} \mathrm{~g} \mathrm{~s}^{-1}$. The 182 identified planets are plotted with the same symbols as in Fig. 1.

as $\mathrm{K}$ type stars and therefore assumed to have large extreme ultraviolet luminosities. The next largest escape rate is found for an M type star, GJ 436 b, for which we estimate a maximum escape rate of the order of $2.2 \times 10^{11} \mathrm{~g} \mathrm{~s}^{-1}$.

For HD 209458b, we obtain an escape rate of $9 \times$ $10^{10} \mathrm{~g} \mathrm{~s}^{-1}$, which is consistent with the lower limit of $\sim 10^{10} \mathrm{~g} \mathrm{~s}^{-1}$ obtained from the interpretation of the Lyman- $\alpha$ transit light curve (Vidal-Madjar et al. 2003; Vidal-Madjar \& Lecavelier des Etangs 2004).

\section{Uncertainties}

For the observed planets, the positions in the previous diagrams are obtained by simple estimates of the EUV luminosities and planetary radii. These rough estimates are subject to uncertainties. These uncertainties add some dispersion in the clouds of points of a given diagram; however with a limited dispersion, this does not severely affect the main trends and the corresponding statistical conclusions, like the empty region observed for lifetime below 5 Gyr. More critically, when we consider a particular planet, we need to have an estimate of the uncertainties introduced by the above approximations.

To evaluate the impact of these approximations on the planetary radius and stellar EUV luminosities, we used the transiting planets. For these planets, we have a measured radius and also a better estimate of the EUV luminosities through the rotational velocity of the central stars $v \sin i$ (when measured; in a few cases only upper limits are available). Because the rotational axis of the stars is expected to be close to the orbital axis of the planets (see for instance Queloz et al. 2000), and for transiting planets $\sin i \cong 1$, the measured $v \sin i$ are in fact true rotational velocities from which better estimates of the EUV luminosities can be obtained. We plotted these transiting planets in the diagram of the potential energy per unit of mass versus EUV energy flux (Fig. 3).

Concerning the radius estimate, OGLE-TR-10b is a special case because this planets has two different radius estimates which are significantly different. From the analysis of Bouchy et al. (2005a), Santos et al. (2006) obtain a radius of 1.43 Jupiter radii, while they obtain a radius of 1.14 Jupiter radii using the

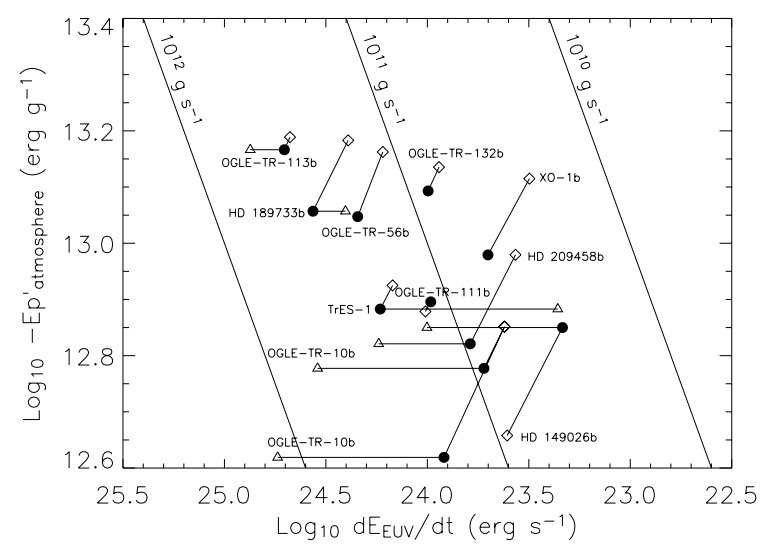

Fig. 3. Potential energy per unit of mass of the atmosphere of the ten transiting planets as a function of the EUV input flux. For each planet the position is calculated using the radius as modeled (diamonds) and the radius as effectively measured (circles). For OGLE-TR-10b we used the two possible planetary radii quoted by Santos et al. (2006). When available, the EUV luminosities estimated from the stellar rotational velocity are plotted with triangles.

new observational data of Holman et al. (2005). Because we cannot discriminate between these results, we keep both radii in the present discussion.

We first evaluate the uncertainty on the radius estimate and its impact on the evaporation rate. Because $\mathrm{d} E_{\mathrm{p}(\mathrm{atm})} / \mathrm{d} m \propto R_{\mathrm{p}}^{-1}$ and $\mathrm{d} E_{\mathrm{EUV}} / \mathrm{d} t \propto R_{\mathrm{p}}^{2}$, the resulting escape rate $\mathrm{d} m / \mathrm{d} t$ is sensitive to the planetary radius and is proportional to $R_{\mathrm{p}}^{3}$. For each planet in Fig. 3, we consider the radius obtained from our fit to Guillot's models (Guillot 2005; plotted with diamonds) and the radius as effectively measured (plotted with filled circles). In the ten transiting planets the difference between the two corresponding escape rates has a mean value of 0.13 dex and a dispersion of 0.22 dex. The largest differences are obtained for OGLE-TR-10 (increase of the escape rate by 0.45 dex with the radius given by Bouchy et al. 2005a), HD 209458 b (increase of the escape rate by 0.33 dex with the real radius) and HD 149026 b (decrease of the escape rate by 0.41 dex with the real radius). This is due to the unexplained excess of planetary radius of OGLE-TR-10 and HD 209458 b (Guillot et al. 2005; Laughlin et al. 2005) and the small radius of HD 189733 b explained by its large metallicity and its suspected massive core (Sato et al. 2005). Although the escape rate is quite sensitive to the radius estimate calculated from the planet characteristics, the transiting planets allow us to estimate that this leads to a typical uncertainty of less than 0.3 dex in the calculated escape rate.

The impact of the EUV energy flux estimate is more difficult to constrain as we do not have real EUV flux measured for any of the stars harboring planets. However, we can use another estimate of the EUV luminosities through true rotational velocity when available in the literature (i.e. for six of the ten transiting planets). For these six planets we calculated the EUV luminosities from their rotational velocity using the results of Wood et al. (1994). Using Rosat S2 measurements of stars within 10 parsecs, Wood et al. (1994) found a close correlation between the S2 luminosity, $L_{\mathrm{S} 2}$, and the stellar rotational velocity, $v_{\text {rot }}: L_{\mathrm{S} 2} \propto v_{\text {rot }}^{1.4}$. From this equation, and assuming $F_{\mathrm{EUV}}=4.6 \mathrm{erg} \mathrm{cm}^{-2} \mathrm{~s}^{-1}$ at $1 \mathrm{AU}$ and $v_{\text {rot }}=2.0 \mathrm{~km} \mathrm{~s}^{-1}$ for the Sun, we find an EUV energy flux of:

$F_{\text {EUV }}(1 \mathrm{AU})=4.6\left(\frac{v_{\text {rot }}}{2.0 \mathrm{~km} \mathrm{~s}^{-1}}\right)^{1.4} \mathrm{erg} \mathrm{cm}^{-2} \mathrm{~s}^{-1}$

at a distance of $1 \mathrm{AU}$ from a star with a rotational velocity $v_{\text {rot }}$. 


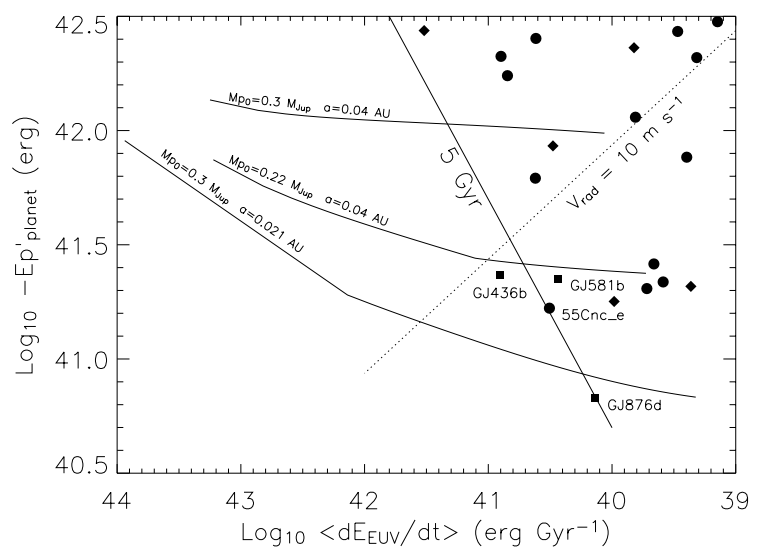

Fig. 4. Potential energy of the extrasolar planets as a function of the EUV input flux with evolutionary tracks superimposed. This is an enlargement of Fig. 1. Time evolution of planets at constant orbital distance $a_{\mathrm{p}}=0.021 \mathrm{AU}$ and $a_{\mathrm{p}}=0.04 \mathrm{AU}$, corresponding to the orbital distances of GJ $876 \mathrm{~d}$ and two Neptune-mass planets (GJ $581 \mathrm{~b}$ and $55 \mathrm{Cnc}$ ), respectively. GJ $581 \mathrm{~b}$ and $55 \mathrm{Cnc}$ e are on the track of a planet with an initial mass $M_{\mathrm{p}}\left(t_{0}\right)=0.22 M_{\text {jup. }}$ GJ $876 \mathrm{~d}$ is on the track of a planet with an initial mass of $M_{\mathrm{p}}\left(t_{0}\right) \sim 0.3 M_{\text {jup }}$, showing that it can be the remnant of a former hot Saturn-mass planet.

For the six transiting planets with published rotational velocity we plot the result in Fig. 3 with triangles. In these six planets the difference between the two corresponding escape rates has a mean value of $0.18 \mathrm{dex}$ and a dispersion of 0.60 dex. The largest differences are obtained for OGLE-TR-10 (increase of the escape rate by 0.82 dex with the EUV luminosity estimated from the rotational velocity) and TrES-1 (decrease of the escape rate by $0.87 \mathrm{dex}$ ). This is due to the large rotational velocity of OGLE-TR-10 with $v \sin i=7.7 \mathrm{~km} \mathrm{~s}^{-1}$ (Bouchy et al. 2005a), and the small rotational velocity of TReS-1 with $v \sin i=1.08 \mathrm{~km} \mathrm{~s}^{-1}$ (Laughlin et al. 2005). As a result, we see that in two of the six cases, we have a large uncertainty of the EUV luminosity with consequently a relatively large uncertainty in the escape rate $(\sim 0.8 \mathrm{dex})$; for the four other cases the dispersion is reduced to 0.4 dex. Taking into account the two uncertainties (radius and energy flux estimates), we find that the differences in the estimated escape rates have a typical dispersion of 0.7 dex.

\section{Evolution tracks}

The diagram described above describes the possibility of the planet evolution on billions years time scale through the evaporation process. The position in the diagram can be calculated or evaluated at a given time, in particular in the present epoch (assuming an age for the parent star). It is thus interesting to see how a planet can evolve in such a diagram. The position in the diagram contains information on the evolution timescale.

We thus calculated evolution tracks for a few cases of planets for which the initial conditions imply rapid evolution through evaporation. This concerns light planets at short distances (Saturn mass planets at very-hot-Jupiter distances). In calculating these evolution tracks, the critical parameter is found to be the planetary radius and mass at a given age, both evolving with time. Again we assumed that the EUV flux varies as $t^{-1}$ and used our fit to the Guillot et al. models and the corresponding time evolution described by Eq. (21). The result is plotted in Fig. 4.

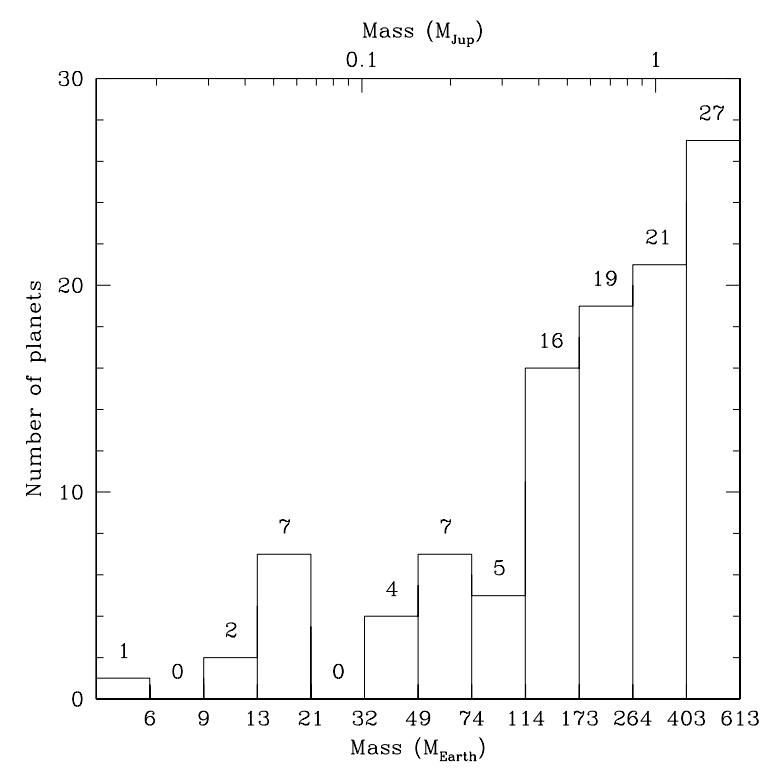

Fig. 5. Mass distribution of planets discovered through radial velocity.

We calculated the time evolution of planets at orbital distance $a_{\mathrm{p}}=0.021 \mathrm{AU}$ and $a_{\mathrm{p}}=0.04 \mathrm{AU}$, corresponding to the orbital distances of GJ $876 \mathrm{~d}$ and two Neptune-mass planets (GJ $581 \mathrm{~b}$ and $55 \mathrm{Cnce}$ ), respectively. We find that GJ $581 \mathrm{~b}$ and $55 \mathrm{Cnc}$ e can be the remnant of a planet with an initial mass of $0.22 M_{\text {jup }}$. The evolution track of a planet with an orbital distance of $0.021 \mathrm{AU}$ and an initial mass of $0.3 M_{\text {jup }}$ shows that GJ $876 \mathrm{~d}$ can be the remnant of a planet whose initial mass could be up to the mass of Saturn.

\section{Neptune mass planets in the diagram}

A plot of the mass distribution of the extrasolar planets shows that Neptune mass planets play a particular role (Fig. 5). Ten planets have been found with mass below $0.067 M_{\text {jup }}(21$ Earthmass), while no planets have been identified with mass between $0.067 M_{\text {jup }}$ and $0.11 M_{\text {jup }}$ (35 Earth-mass). The presence of this gap is not related to a bias in the radial velocity searches, since more massive planets are easier to detect (at the same orbital distance). This reveals the different nature of these Neptune mass planets orbiting at short orbital distances. Their nature is still a matter of debate (Baraffe et al. 2005). They may be the remnants of evaporated more massive planets ("chthonian planets") as foreseen in Lecavelier des Etangs et al. (2004). Other possibilities include gaseous Neptune-like planets, super-Earth (Santos et al. 2004) or ocean-planets (Kutchner 2003; Léger et al. 2004).

The mass of these objects being measured, the freedom of their position in the energy diagram mainly relies on the determination of their radius, or, in other words, of their mean density, i.e., their nature. As a first approach, in Fig. 1 we considered that these planets have a typical density of $2 \mathrm{~g} \mathrm{~cm}^{-3}$ for 15 Earth mass and $6 \mathrm{~g} \mathrm{~cm}^{-3}$ for 6 Earth mass. Now we can test the density hypothesis and determine the possible nature of these planets. We plotted the position of these Neptune mass planets in the energy diagram with different hypotheses about their density (Fig. 6). We used a mean planetary density of $\rho_{\mathrm{p}}=6 \mathrm{~g} \mathrm{~cm}^{-3}$ for a typical density of refractory-rich planets, which should describe the chthonian and super-Earth planets. A lower density, of the order of $\rho_{\mathrm{p}}=2 \mathrm{~g} \mathrm{~cm}^{-3}$, can be considered as more plausible for volatile-rich planets such as the ocean planets. For gas-rich planets we assumed a much lower density of $\rho_{\mathrm{p}}=0.2 \mathrm{~g} \mathrm{~cm}^{-3}$ 


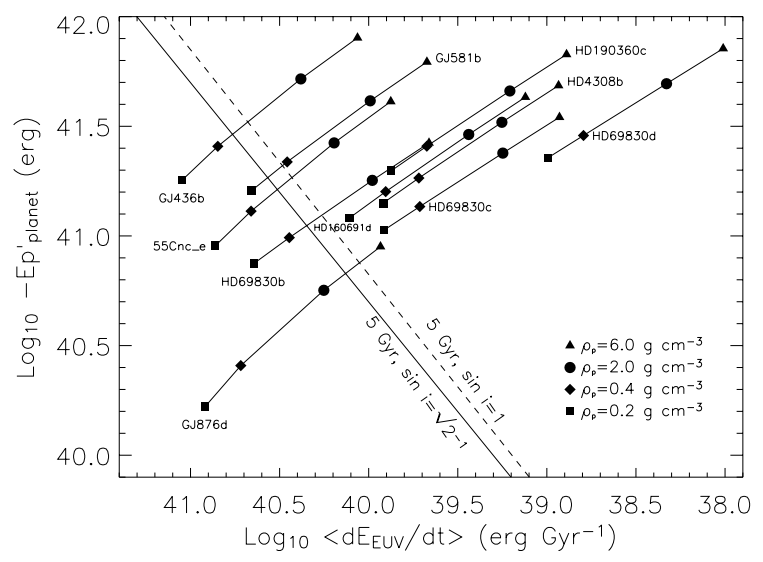

Fig. 6. Plot of the potential energy of the Neptune mass planet as a function of the EUV flux for various planets' density. For GJ 581 b, GJ 436 b, HD $69830 \mathrm{~b}, 55 \mathrm{Cnce}$ and GJ $876 \mathrm{~d}$, and assuming $\sin i=\sqrt{2^{-1}}$, lifetime shorter than $5 \mathrm{Gyr}$ are obtained for densities below $0.28,0.55$, $0.56,0.69$ and $3.1 \mathrm{~g} \mathrm{~cm}^{-3}$, respectively. If $\sin i=1$ (dotted line), the critical (minimum) densities are increased to 0.38, 0.74, 0.78, 0.93 and $4.2 \mathrm{~g} \mathrm{~cm}^{-3}$, for GJ $581 \mathrm{~b}, \mathrm{GJ} 436 \mathrm{~b}, \mathrm{HD} 69830 \mathrm{~b}, 55 \mathrm{Cnc}$, and GJ 876 respectively.

and $\rho_{\mathrm{p}}=0.4 \mathrm{~g} \mathrm{~cm}^{-3}$, describing planets that look more like irradiated Neptune-like planets (Baraffe et al. 2006, find densities of 0.1 to $0.25 \mathrm{~g} \mathrm{~cm}^{-3}$ for the final stage of an evaporated gaseous planets with a 6 Earth mass core and final mass of about one Neptune mass).

In the energy diagram, HD $190360 \mathrm{c}, \mathrm{HD} 4308 \mathrm{~b}$, HD $160691 \mathrm{~d}$, HD 69830 c and HD 69830d are all located in the possible domain with lifetimes above $5 \mathrm{Gyr}$, independent of their assumed density. Even with very low densities, down to $0.2 \mathrm{~g} \mathrm{~cm}^{-3}$, these planets do not receive enough energy from their central star to evaporate significantly. For GJ $581 \mathrm{~b}$ only the extreme case $\rho_{\mathrm{p}}=0.2 \mathrm{~g} \mathrm{~cm}^{-3}$ is in the evaporation-forbidden region. Due to their orbital distances, between 0.09 and $0.63 \mathrm{AU}$, it appears that the evaporation is not efficient enough to modify the nature of these planets. These planets are not likely to be the evaporation remnants of previously more massive planets

For GJ 436b, 55 Cnce and HD 69830b, a density below $0.4 \mathrm{~g} \mathrm{~cm}^{-3}$ would place these planets in the evaporationforbidden region of the energy diagram. If these planets were formerly low density (gaseous giant) planets, they must have lost a large fraction of their upper atmospheric gas. We therefore conclude that, with low orbital distances in the range 0.03 to $0.08 \mathrm{AU}$, these planets cannot belong to the category of low mass gaseous planet (irradiated Neptune-like planets). The position of these planets in the energy diagram shows that they must contain a large fraction of solid/liquid material in the form of volatile or refractory elements. These planets can be the remnants of previously more massive planets (chthonian planets) or volatile-rich planets (ocean planets) or rocky planets (superEarths).

The conclusion for HD 69830 b is consistent with the one reached by Lovis et al. (2006) who concluded from formation and migration models that it must have a mainly rocky composition.

For GJ $876 \mathrm{~d}$, even the density of an ocean planet creates some problems. GJ $876 \mathrm{~d}$ needs to be a planet dense enough to be located above the $t_{2}=5 \mathrm{Gyr}$ lifetime limit. This planet requires a density higher than $3.1 \mathrm{~g} \mathrm{~cm}^{-3}$ for its atmosphere to survive. GJ $876 \mathrm{~d}$ could be a big rocky planet, like a super-Earth, or a refractory remnant of a previously more massive planet (an evaporated ocean planet?). However, its parent star is an $M$ star, and we have very little information about the EUV flux of non-active M dwarfs; even if every $\mathrm{M}$ star has period of strong activity remains unclear. A more detailed analysis of the energy flux from this star and its possible consequences on this intriguing planet is needed.

Thus, the energy diagram allows us to trace three different categories for the ten presently identified Neptune mass planets. For six of them, the EUV input energy seems not strong enough to significantly affect these planets; we cannot conclude on their nature. For three other planets, it appears that they cannot be low mass gaseous planets. These planets must contain a large fraction of solid/liquid material. Finally, GJ $876 \mathrm{~d}$ must be dense enough to survive the strong EUV energy flux from its nearby parent star. This planet must contain a large fraction of massive elements.

\section{Conclusion}

To describe the evaporation status of the extrasolar planets, we consider an energy diagram in which the potential energy of the planets is plotted versus the energy received by the upper atmosphere. We presented a basic method to estimate these quantities. For the energy flux, we proposed a first approach using the type of the parent stars or, if available, their rotation period. For the potential energy, we included the modification of the gravity field by the tidal forces from the parent stars. This description allows a rapid estimate of both the escape rate of the atmospheric gas and the lifetime of a planet against the evaporation process. We found that the region of lifetime below $5 \mathrm{Gyr}$ is devoid of planets, which reinforces the belief that this diagram is an appropriate description of evaporation. In the evaporationforbidden region, the evaporation can modify the nature and the characteristics of the planets such that they move to a region of the diagram corresponding to longer lifetimes.

The escape rates estimated from the diagram are found to be in the range $10^{5} \mathrm{~g} \mathrm{~s}^{-1}$ to $10^{12} \mathrm{~g} \mathrm{~s}^{-1}$, with a few cases above $10^{11} \mathrm{~g} \mathrm{~s}^{-1}$. Although this is a crude estimate, the estimated escape rate for HD 209458 b is found to be consistent with the lower limit of $10^{10} \mathrm{~g} \mathrm{~s}^{-1}$ obtained from interpretation of the H I Lyman- $\alpha$ observations.

This diagram allows the description of the possible nature of the recently discovered Neptune-mass planets. We found that GJ 436 b, 55 Cnce and HD 69830 b cannot be low mass gaseous planets. These planets must contain a large fraction of solid/liquid material. We argued that GJ $876 \mathrm{~d}$ must be dense enough to survive the strong EUV energy flux from its nearby parent star. GJ $876 \mathrm{~d}$ must contain a large fraction of massive elements.

Acknowledgements. This work has been motivated by many discussions with various people. In particular, I am extremely grateful to F. Bouchy, G. Hébrard, J. McConnell, F. Pont, G. Tinetti and A. Vidal-Madjar. I warmly thank the anonymous referee for detailed comments which helped to clarify the paper. I thank the International Space Science Institute (ISSI) in Berne, Swizerland, for support and discussion during the workshop on transiting extrasolar planets.

\section{References}

Baraffe, I., Selsis, F., Chabrier, G., et al. 2004, A\&A, 419, L13 Baraffe, I., Chabrier, G., Barman, T. S., et al. 2005, A\&A, 436, L47 Baraffe, I., Alibert, Y., Chabrier, G., \& Benz, W. 2006, A\&A, 450, 1221 Bouchy, F., Pont, F., Melo, C., et al. 2005a, A\&A, 431, 1105 Bouchy, F., Udry, S., Mayor, M., et al. 2005b, A\&A, 444, L15 Burrows, A., \& Lunine, J. 1995, Nature, 378, 333

Burrows, A., Guillot, T., Hubbard, W. B., et al. 2000, ApJ, 534, L97 
Chamberlain, J. W., \& Hunten, D. M. 1987, in Theory of planetary atmospheres (Orlando: Acad. Press), Int. Geophys. Ser., 36

García Muñoz, A. 2006, Icarus, submitted

Gaudi, B. S., Seager, S., \& Mallen-Ornelas, G. 2005, ApJ, 623, 472

Guillot, T. 2005, Annual Review of Earth and Planetary Sciences, 33, 493

Guillot, T., Burrows, A., Hubbard, W. B., Lunine, J. I., \& Saumon, D. 1996, ApJ, 459, L35

Guillot, T., Santos, N. C., Pont, F., et al. 2006, A\&A, 453, L21

Guinan, E. F., Ribas, I., Harper, G. M., \& Guedel, M. 2002, BAAS, 34, 726

Hodgkin, S. T., \& Pye, J. P. 1994, MNRAS, 267, 840

Holman, M. J., Winn, J. N., Stanek, K. Z., et al. 2005

[arXiv:astro-ph/0506569]

Kuchner, M. J. 2003, ApJ, 596, L105

Kurucz, R. 1993, ATLAS9 Stellar Atmosphere Programs and 2 km/s grid, CD-ROM No. 13

Lakatos, S. L., Voyer, E. N., Guinan, E. F., et al. 2005, Am. Astron. Soc. Meet. Abstracts, 206, \#09.03

Laughlin, G., Wolf, A., Vanmunster, T., et al. 2005, ApJ, 621, 1072

Lecavelier des Etangs, A., \& Vidal-Madjar, A. 2006, in Planet Formation: Theory, Observation and Experiments, ed. H. Klahr, \& W. Brandner, Chap. 9, 147

Lecavelier des Etangs, A., Vidal-Madjar, A., Hébrard, G., \& McConnell, J. 2004, A\&A, 418, L1

Léger, A., Selsis, F., Sotin, C., et al. 2004, Icarus, 169, 499
McCullogh, P., Stys, J., Valenti, J., et al. 2006, ApJ, in press [arXiv:astro-ph/0605414]

Mayor, M., \& Queloz, D. 1995, Nature, 378, 355

de Pater, I., \& Lissauer, J. J. 2001, in Planetary Sciences (Cambridge University Press)

Queloz, D., Eggenberger, A., Mayor, M., et al. 2000, A\&A, 359, L13

Ribas, I., Guinan, E. F., Güdel, M., \& Audard, M. 2005, ApJ, 622, 680

Rivera, E. J., Lissauer, J. J., Butler, R. P., et al. 2005, ApJ, 634, 625

Saffe, C., Gómez, M., \& Chavero, C. 2005, A\&A, 443, 609

Santos, N. C., Bouchy, F., Mayor, M., et al. 2004, A\&A, 426, L19

Santos, N. C., Pont, F., Melo, C., et al. 2006, A\&A, 450, 825

Sato, B., Fischer, D. A., Henry, G. W., et al. 2005, ApJ, 633, 465

Schneider, J. 2006, http : //www . obspm. fr/planets

Tian, F., Toon, O. B., Pavlov, A. A., \& De Sterck, H. 2005, ApJ, 621, 1049

Valenti, J. 2005, HST Proposal, \#10718

Vardavas, I. M. 2005, MNRAS, 363, L51

Vidal-Madjar, A., \& Lecavelier des Etangs, A. 2004, Extrasolar Planets: Today and Tomorrow, ASP Conf. Ser., 321, 152

Vidal-Madjar, A., Lecavelier des Etangs, A., Désert, J.-M., et al. 2003, Nature, 422,143

Vidal-Madjar, A., Désert, J.-M., Lecavelier des Etangs, A., et al. 2004, ApJ, 604, L69

Yelle, R. V. 2004, Icarus, 170, 167

Wood, B. E., Brown, A., Linsky, J. L., et al. 1994, ApJS, 93, 287 
A. Lecavelier des Etangs: A diagram to determine the evaporation status of extrasolar planets, Online Material $p 1$

\section{Online Material}




\section{Appendix A: The potential energy of a gaseous planet}

The potential energy of a gaseous sphere of radius $R_{\mathrm{p}}$ is given by

$E_{\mathrm{p}}=-G \int_{0}^{R_{\mathrm{p}}} \frac{m(r)}{r} \frac{\mathrm{d} m(r)}{\mathrm{d} r} \mathrm{~d} r$

where $G$ is the gravitational constant and $m(r)$ is the mass inside a radius $r$. The internal structure, characterized by $m(r)$, is given by

$\frac{\mathrm{d} P}{\mathrm{~d} r}=-\rho(r) \frac{G m(r)}{r^{2}}$,

where $\rho(r)$ is the density given by

$\frac{\mathrm{d} m}{\mathrm{~d} r}=4 \pi r^{2} \rho(r)$,

and the relation between the pressure $P(r)$ and the density $\rho(r)$ is given by the equation of state. An analytical solution can be found if we assume that the equation of state follows a polytrope with an index $n=1$, which is found to be a good approximation for the gaseous planets interior (de Pater \& Lissauer 2001). In that case, the equation of state is

$P=K \rho^{2}$,

where $K$ is a constant. Equations (A.2)-(A.4) can be analytically solved. This gives the density profile within the planet (de Pater \& Lissauer 2001):

$\rho(r)=\rho_{c}\left(\frac{\sin \left(C_{K} r\right)}{C_{K} r}\right)$,

with

$C_{K}=\frac{\pi}{R_{\mathrm{p}}}=\sqrt{\frac{2 \pi G}{K}}$.

Putting Eq. (A.5) into Eq. (A.1), this gives

$E_{\mathrm{p}}=-\frac{12 G \pi^{3} \rho_{c}^{2}}{C_{K}^{5}}$

Also using Eqs. (A.3), (A.5) and (A.6), the planet mass, $M_{\mathrm{p}}$ is given by

$M_{\mathrm{p}}=m\left(R_{\mathrm{p}}\right)=m\left(\pi / C_{K}\right)=\frac{4 \pi^{2} \rho_{c}}{C_{K}^{3}}$.

Finally, putting Eqs. (A.6) and (A.8) into (A.7) we find the potential energy of a gaseous sphere in which the equation of state follows a polytropic with an index $n=1$ :

$E_{\mathrm{p}}=-\frac{3}{4} G M_{\mathrm{p}}^{2} / R_{\mathrm{p}}$

\section{Appendix B: Modification of the potential energy by tidal forces}

In the field of a planet of mass $M_{\mathrm{p}}$, at a distance $r$ from the planet center, the potential energy field, $\chi$, follows the equation

$\chi(r)=-\frac{G M_{\mathrm{p}}}{r}+C$,

where $G$ is the gravitational constant and $C$ is a constant. The potential energy of a unit mass of the atmospheric gas of a planet, $\mathrm{d} E_{\mathrm{p}(\mathrm{atm})} / \mathrm{d} m$, can be defined by the opposite of the energy needed to escape the planet gravity, in other words to reach infinite distance from the planet:

$\mathrm{d} E_{\mathrm{p}(\mathrm{atm})} / \mathrm{d} m \equiv \chi\left(R_{\mathrm{p}}\right)-\chi(\infty)=-G M_{\mathrm{p}} / R_{\mathrm{p}}$,

where $R_{\mathrm{p}}$ is the radius of the planet. This demonstrates the well known Eq. (6) given in Sect. 4.2.

However, in a star-planet system, the potential energy field is modified by the star gravity and centrifugal forces. Along the star-planet axis, the potential energy field, $\chi^{\prime}$, follows the equation:

$$
\begin{aligned}
\chi^{\prime}(r)= & \chi(r)+\Delta \chi(r) \\
= & -G\left(M_{\mathrm{p}}+M_{*}\right)\left[\frac{\mu}{r}+\frac{1-\mu}{a_{\mathrm{p}}-r}+\frac{\left[(1-\mu) a_{\mathrm{p}}-r\right]^{2}}{2 a_{\mathrm{p}}^{3}}\right] \\
& +C,
\end{aligned}
$$

where $r$ is the distance from the planet center in the direction of the star on the star-planet axis, $a_{\mathrm{p}}$ is the star-planet distance, $M_{*}$ is the stellar mass and $\mu$ is defined by

$\mu=\frac{M_{\mathrm{p}}}{M_{\mathrm{p}}+M_{*}}$.

In the case $r \ll a_{\mathrm{p}}$ and $\mu \ll 1$, we can derive

$$
\frac{\Delta \chi(r)}{G\left(M_{\mathrm{p}}+M_{*}\right)}=-\frac{3}{2 a_{\mathrm{p}}}\left(1+\frac{r^{2}}{a_{\mathrm{p}}^{2}}+O\left(\frac{r^{3}}{a_{\mathrm{p}}^{3}}\right)+O(\mu)\right)+C .
$$

In the modified gravity field, the energy needed to escape the planet can be defined by the energy needed to reach the Roche lobe. Along the star-planet axis, the Roche lobe is located at a distance $r=r_{\text {Roche }}$, where $r_{\text {Roche }}$ follows:

$\frac{\partial \chi^{\prime}}{\partial r}\left(r_{\text {Roche }}\right)=0$

Again assuming that $r \ll a_{\mathrm{p}}$, and using Eqs. (B.3) and (B.6) we find

$\mu=3\left(\frac{r_{\text {Roche }}}{a_{\mathrm{p}}}\right)^{3}+O\left(\frac{r_{\text {Roche }}}{a_{\mathrm{p}}}\right)^{4}$,

from which we derive

$\frac{r_{\text {Roche }}}{a_{\mathrm{p}}} \approx\left(\frac{\mu}{3}\right)^{1 / 3}$.

Finally, the new potential energy of a unit mass of the atmospheric gas of a planet, $\mathrm{d} E_{\mathrm{p}(\mathrm{atm})}^{\prime} / \mathrm{d} m$, defined by the opposite of the energy needed to escape the planet gravity, is now the energy to reach the Roche lobe:

$$
\begin{aligned}
\mathrm{d} E_{\mathrm{p}(\mathrm{atm})}^{\prime} / \mathrm{d} m \equiv & \chi^{\prime}\left(R_{\mathrm{p}}\right)-\chi^{\prime}\left(r_{\text {Roche }}\right) \\
= & {\left[\chi\left(R_{\mathrm{p}}\right)-\chi\left(r_{\text {Roche }}\right)\right] } \\
& +\left[\Delta \chi\left(R_{\mathrm{p}}\right)-\Delta \chi\left(r_{\text {Roche }}\right)\right]
\end{aligned}
$$

In the case $\mu \ll 1$ and $R_{\mathrm{p}} \ll a_{\mathrm{p}}$, using Eqs. (B.1) and (B.7) we derive for the first term of Eq. (B.9):

$$
\begin{aligned}
\frac{\chi\left(R_{\mathrm{p}}\right)-\chi\left(r_{\text {Roche }}\right)}{G\left(M_{\mathrm{p}}+M_{*}\right)}= & -\frac{\mu}{R_{\mathrm{p}}}+\frac{3}{a_{\mathrm{p}}}\left(\frac{r_{\text {Roche }}}{a_{\mathrm{p}}}\right)^{2} \\
& +\frac{1}{a_{\mathrm{p}}} O\left(\frac{r_{\text {Roche }}}{a_{\mathrm{p}}}\right)^{3} .
\end{aligned}
$$


For the second term of Eq. (B.9), using Eq. (B.5) we derive:

$$
\begin{aligned}
& \frac{\Delta \chi\left(R_{\mathrm{p}}\right)-\Delta \chi\left(r_{\text {Roche }}\right)}{G\left(M_{\mathrm{p}}+M_{*}\right)}=\frac{3}{2 a_{\mathrm{p}}}\left(\frac{r_{\text {Roche }}}{a_{\mathrm{p}}}\right)^{2} \\
& +\frac{1}{a_{\mathrm{p}}}\left(O\left(\frac{R_{\mathrm{p}}}{a_{\mathrm{p}}}\right)^{2}+O\left(\frac{r_{\text {Roche }}}{a_{\mathrm{p}}}\right)^{3}+O(\mu)\right) .
\end{aligned}
$$

Here we implicitly assumed $\left(r_{\text {Roche }} / R_{\mathrm{p}}\right)^{2} \gg 1$, which is valid as the smallest values in extreme cases of very-hot-Jupiters are always larger than 10 .

As a result, combining Eqs. (B.2), (B.9), (B.10) and (B.11) we find

$$
\begin{aligned}
\mathrm{d} E_{\mathrm{p}(\mathrm{atm})}^{\prime} / \mathrm{d} m \approx & \mathrm{d} E_{\mathrm{p}(\mathrm{atm})} / \mathrm{d} m \\
& +\frac{9 G\left(M_{\mathrm{p}}+M_{*}\right)}{2 a_{\mathrm{p}}}\left(\frac{r_{\text {Roche }}}{a_{\mathrm{p}}}\right)^{2} .
\end{aligned}
$$

If we define $\Delta_{\text {tidal }} \mathrm{d} E_{\mathrm{p}(\mathrm{atm})} / \mathrm{d} m$ by

$\mathrm{d} E_{\mathrm{p}(\mathrm{atm})}^{\prime} / \mathrm{d} m \equiv \mathrm{d} E_{\mathrm{p}(\mathrm{atm})} / \mathrm{d} m+\Delta_{\text {tidal }} \mathrm{d} E_{\mathrm{p}(\mathrm{atm})} / \mathrm{d} m$,

using Eqs. (B.8) and (B.12), we find

$\Delta_{\text {tidal }} \mathrm{d} E_{\mathrm{p}(\mathrm{atm})} / \mathrm{d} m \approx \frac{3^{4 / 3} G\left(M_{\mathrm{p}}+M_{*}\right) \mu^{2 / 3}}{2 a_{\mathrm{p}}}$.

That is

$\Delta_{\text {tidal }} \mathrm{d} E_{\mathrm{p}(\mathrm{atm})} / \mathrm{d} m \approx \frac{3^{4 / 3}}{2} \frac{G M_{*}^{1 / 3} M_{\mathrm{p}}^{2 / 3}}{a_{\mathrm{p}}}$.

This result is obtained using the potential description in the direction of the star on the star-planet axis, but it is valid in all directions because the potential is the same on the whole Roche lobe surface which is defined as an iso-potential. Therefore the energy needed to reach the Roche lobe and to escape the planet is the same in all directions.

\section{Appendix C: The radial velocity iso-curves in the energy diagram}

Most of the known planets have been detected with the radial velocity method which is not sensitive to low mass planets with large orbital distances. Therefore, if the detected planets are plotted in the energy diagram (Fig. 1), the absence of planets with small absolute values of the potential energy (low mass) receiving a low EUV energy (large orbital distance) is due to this bias of the radial velocity method. The amplitude of the radial velocity of a planet-hosting star, $V_{\text {rad }}$, is given by

$V_{\mathrm{rad}}^{2}=\frac{G M_{\mathrm{p}}^{2}}{a_{\mathrm{p}} M_{*}}$, where $G$ is the gravitational constant, $a_{\mathrm{p}}$ the planet orbital distance, $M_{\mathrm{p}}$ and $M_{*}$ are the mass of the planet and the star, respectively. Using Eq. (4), and the fact that

$\frac{\mathrm{d} E_{\mathrm{EUV}} / \mathrm{d} t}{P_{*, \mathrm{EUV}}}=\frac{R_{\mathrm{p}}^{2}}{4 a_{\mathrm{p}}^{2}}$,

where $P_{* \text { EUV }}$ is the total power emitted in EUV by the parent star, we derive

$E_{\mathrm{p}} \approx-\frac{3}{8} M_{*} V_{\mathrm{rad}}^{2} \sqrt{\left(\frac{\mathrm{d} E_{\mathrm{EUV}} / \mathrm{d} t}{P_{*, \mathrm{EUV}}}\right)^{-1}}$.

This equation does not depend on the planetary radius, mass and orbital distance. Therefore, for a given stellar type, corresponding to a given stellar mass and total power emitted in the EUV, the radial velocity iso-curves must follow a straight line in the $\log -\log$ energy diagram of $E_{\mathrm{p}}$ as a function of $\mathrm{d} E_{\mathrm{EUV}} / \mathrm{d} t$.

Most of the instruments have a detection threshold at about $10 \mathrm{~m} \mathrm{~s}^{-1}$. Instruments with higher sensitivity (like the Harps spectrograph) are recent and allowed the discovery of low mass planets only with short orbital periods. This explains why the detected planets below the limit at $10 \mathrm{~m} \mathrm{~s}^{-1}$ are in the left part of the energy diagram plotted in Fig. 1. 